\title{
TWO-PARTICLE SYSTEM WITH HARMONIC OSCILLATOR INTERACTION IN NONCOMMUTATIVE PHASE SPACE
}

\author{
Kh. P. Gnatenko, V. M. Tkachuk \\ Ivan Franko National University of Lviv, Department for Theoretical Physics, \\ 12, Drahomanov St., Lviv, 79005, Ukraine, \\ e-mail:khrystyna.gnatenko@gmail.com,voltkachuk@gmail.com \\ (Received June 21, 2017; in final form - July 26, 2017)
}

\begin{abstract}
A two-particle system is studied in a space with noncommutativity of coordinates and noncommutativity of momenta. We consider the case when different particles feel noncommutativity with different parameters, which are determined by their masses. We have found exactly the spectrum of a two-particle system with harmonic oscillator interaction in the noncommutative phase space.

Key words: noncommutative phase space, two-particle system, harmonic oscillator.

PACS number(s): 03.65.-w, 02.40.Gh
\end{abstract}

\section{INTRODUCTION}

The idea of noncommutativity is quite old. It was proposed by Heisenberg. The physicist told the idea to Peierls, who informed Pauli about it. Pauli told the idea to Oppenheimer. Oppenheimer asked his student Snyder to work on the subject [1]. The results of his work were published in paper [2]. For a long time, the idea of noncommutativity was not being studied intensively. In recent years because of the development of the String Theory and Quantum Gravity (see, for example, $[3,4])$ the interest in the studies of noncommutativity has risen significantly. Different problems were studied in the framework of different types of noncommutativity, among them the harmonic oscillator [5-13], the hydrogen atom [10, 14-28], the Landau problem [29-32], the gravitational quantum well $[33,34]$, classical systems with various potentials [35-40], many-particle systems [10, 14, 42-46], and many others.

In the four-dimensional noncommutative phase space (2D configurational space and $2 \mathrm{D}$ momentum space), the coordinates and momenta satisfy the following commutation relations

$$
\begin{aligned}
& {\left[X_{1}, X_{2}\right]=i \hbar \theta} \\
& {\left[X_{i}, P_{j}\right]=i \hbar \delta_{i j}} \\
& {\left[P_{1}, P_{2}\right]=i \hbar \eta}
\end{aligned}
$$

Note that in contrast to the ordinary space, in the noncommutative phase space the coordinates and momenta do not commute (1), (3). The parameters $\theta, \eta$ are constants and are called the parameters of noncommutativity, $i=(1,2), j=(1,2)$.

In the general case, different particles may feel noncommutativity with different parameters. Therefore there is the problem of describing the motion of the center-of-mass of a system made of $N$ particles in the noncommutative phase space. This problem was studied in our previous paper [40]. On the basis of the results presented in [40] in the present paper we study the particular case when a composite system consists of two par- ticles with harmonic oscillator interaction. We examine the influence of noncommutativity on the energy levels of the system, which are found exactly.

The article is organized as follows. Section II discusses the problem of describing the two-particle motion in the four-dimensional noncommutative phase space. In Section III, we calculate exactly the energy levels of a two-particle system with harmonic oscillator interaction. Conclusions are presented in Section IV.

\section{REDUCTION OF THE TWO-PARTICLE PROBLEM TO A ONE-PARTICLE PROBLEM IN THE NONCOMMUTATIVE PHASE SPACE}

Let us consider a two-particle system which consists of particles having masses $m_{1}$ and $m_{2}$ in a four-dimensional noncommutative phase space and study the following Hamiltonian

$$
H=\frac{\left(\mathbf{P}^{(1)}\right)^{2}}{2 m_{1}}+\frac{\left(\mathbf{P}^{(2)}\right)^{2}}{2 m_{2}}+U\left(\left|\mathbf{X}^{(1)}-\mathbf{X}^{(2)}\right|\right),
$$

with $U\left(\left|\mathbf{X}^{(1)}-\mathbf{X}^{(2)}\right|\right)$ being the interaction potential energy. The coordinates $X_{i}^{(a)}$ and the momenta $P_{i}^{(a)}$ of a particle satisfy the following relations

$$
\begin{aligned}
& {\left[X_{1}^{(a)}, X_{2}^{(b)}\right]=i \hbar \delta^{a b} \theta_{a},} \\
& {\left[X_{i}^{(a)}, P_{j}^{(b)}\right]=i \hbar \delta^{a b} \delta_{i j},} \\
& {\left[P_{1}^{(a)}, P_{2}^{(b)}\right]=i \hbar \delta^{a b} \eta_{a},}
\end{aligned}
$$

here $\theta_{a}, \eta_{a}$ are the parameters of noncommutativity which correspond to a particle of mass $m_{a}, i=(1,2)$, $j=(1,2)$.

Hamiltonian (4) can be represented in the following form

$$
H=\frac{(\tilde{\mathbf{P}})^{2}}{2 M}+\frac{(\Delta \mathbf{P})^{2}}{2 \mu}+U(|\Delta \mathbf{X}|)
$$


where we use the following notations for the momenta and coordinates of the center-of-mass, the momenta and coordinates of the relative motion,

$$
\begin{aligned}
& \tilde{\mathbf{P}}=\mathbf{P}^{(1)}+\mathbf{P}^{(2)} \\
& \tilde{\mathbf{X}}=\frac{m_{1} \mathbf{X}^{(1)}+m_{2} \mathbf{X}^{(2)}}{m_{1}+m_{2}} \\
& \Delta \mathbf{P}=\mu_{1} \mathbf{P}^{(2)}-\mu_{2} \mathbf{P}^{(1)} \\
& \Delta \mathbf{X}=\mathbf{X}^{(2)}-\mathbf{X}^{(1)}
\end{aligned}
$$

where $M=m_{1}+m_{2}$ is the total mass and $\mu=$ $m_{1} m_{2} /\left(m_{1}+m_{2}\right)$ is the reduced mass, $\mu_{a}=m_{a} / M$. Note that the coordinates $X_{i}^{(a)}$ and the momenta $P_{i}^{(a)}$ in (9)-(12) satisfy (5)-(7).

It is important to stress that because of noncommutativity, the motion of the center-of-mass and the relative motion are not independent in the noncommutative phase space [40]. Taking into account (5)-(7), (9)-(12), one can obtain the following commutation relations for the coordinates and momenta of the center-of-mass and the coordinates and momenta of the relative motion

$$
\begin{aligned}
& {\left[\tilde{X}_{1}, \tilde{X}_{2}\right]=i \hbar \tilde{\theta},} \\
& {\left[\tilde{P}_{1}, \tilde{P}_{2}\right]=i \hbar \tilde{\eta},} \\
& {\left[\tilde{X}_{i}, \tilde{P}_{j}\right]=\left[\Delta X_{i}, \Delta P_{j}\right]=i \hbar \delta_{i j},} \\
& {\left[\Delta X_{1}, \Delta X_{2}\right]=i \hbar \Delta \theta,} \\
& {\left[\Delta P_{1}, \Delta P_{2}\right]=i \hbar \Delta \eta,} \\
& {\left[\tilde{P}_{1}, \Delta P_{2}\right]=-\left[\tilde{P}_{2}, \Delta P_{1}\right]=i \hbar\left(\mu_{1} \eta_{2}-\mu_{2} \eta_{1}\right),} \\
& {\left[\tilde{X}_{1}, \Delta X_{2}\right]=i \hbar\left(\mu_{2} \theta_{2}-\mu_{1} \theta_{1}\right) ;}
\end{aligned}
$$

here

$$
\begin{aligned}
& \tilde{\theta}=\mu_{1}^{2} \theta_{1}+\mu_{2}^{2} \theta_{2}, \\
& \tilde{\eta}=\eta_{1}+\eta_{2}, \\
& \Delta \theta=\theta_{1}+\theta_{2}, \\
& \Delta \eta=\mu_{2}^{2} \eta_{1}+\mu_{1}^{2} \eta_{2}
\end{aligned}
$$

are the effective parameters of noncommutativity. Note that because of noncommutativity (18), (19) the twoparticle problem can not be reduced to a one-particle problem in noncommutative phase space.

In our papers $[40,41]$, we proposed conditions on the parameters of noncommutativity

$$
\begin{aligned}
& \frac{\eta_{a}}{m_{a}}=\alpha=\mathrm{const}, \\
& \theta_{a} m_{a}=\gamma=\mathrm{const}
\end{aligned}
$$

with $\alpha, \gamma$ being constants which are the same for particles with different masses, $\theta_{a}, \eta_{a}$ are the parameters of noncommutativity which correspond to a particle with mass $m_{a}$. Note that in (24), (25) there is no summation. The conditions give the possibility to solve a list of problems in the noncommutative phase space. Among them are the problem of the dependence of the motion of the center-of-mass of a composite system on the relative motion; the problem of the violation of the equivalence principle; the problem of the violation of properties of the kinetic energy. We would also like to note here that a similar condition on the parameter of deformation $\sqrt{\beta} m=\gamma=$ const is important in a space with minimal length $[\hat{X}, \hat{P}]=i \hbar\left(1+\beta \hat{P}^{2}\right)$. As was shown in [47-49], the equivalence principle is recovered, the properties of the kinetic energy are preserved, the Galilean and Lorentz transformations do not depend on mass if this condition holds.

It is clear that commutators (18), (19) are equal to zero when relations (24), (25) are satisfied. Therefore,

$$
\left[\frac{(\tilde{\mathbf{P}})^{2}}{2 M}, \frac{(\Delta \mathbf{P})^{2}}{2 \mu}+U(|\Delta \mathbf{X}|)\right]=0
$$

and the two-particle problem can be reduced to the problems of the motion of the center-of-mass and the relative motion, which can be studied separately.

\section{THE ENERGY LEVELS OF A TWO-PARTICLE SYSTEM WITH HARMONIC OSCILLATOR INTERACTION}

Let us study a particular case when a system consists of two particles with harmonic oscillator interaction

$$
U\left(\left|\mathbf{X}^{(1)}-\mathbf{X}^{(2)}\right|\right)=\frac{k}{2}\left(\mathbf{X}^{(1)}-\mathbf{X}^{(2)}\right)^{2},
$$

here $k$ is a constant. In this case the Hamiltonian of the system reads

$$
H=\frac{\left(\mathbf{P}^{(1)}\right)^{2}}{2 m_{1}}+\frac{\left(\mathbf{P}^{(2)}\right)^{2}}{2 m_{2}}+\frac{k}{2}\left(\mathbf{X}^{(1)}-\mathbf{X}^{(2)}\right)^{2} .
$$

Using (9)-(12), the Hamiltonian can be rewritten as

$$
H=H_{\mathrm{c}}+H_{\mathrm{rel}},
$$

where the Hamiltonians

$$
\begin{aligned}
& H_{\mathrm{c}}=\frac{\tilde{\mathbf{P}}^{2}}{2 M}, \\
& H_{\mathrm{rel}}=\frac{\Delta \mathbf{P}^{2}}{2 \mu}+\frac{k}{2} \Delta \mathbf{X}^{2},
\end{aligned}
$$

describe the motion of the center-of-mass and the relative motion, respectively, $M=m_{1}+m_{2}, \mu=m_{1} m_{2} /\left(m_{1}+\right.$ $\left.m_{2}\right)$. Let us find the energy levels of a two-particle system with harmonic oscillator interaction in noncommutative phase space. As was shown in the previous section, the motion of the center-of-mass and the relative motion are independent if conditions (24), (25) hold. So, in this case we can consider eigenvalue problems for $H_{\text {rel }}$ and $H_{\mathrm{c}}$ independently.

First, let us study the motion of the center-of-mass described by $H_{\mathrm{c}}$. Note that the total momenta $\tilde{P}_{i}$ satisfy commutation relations (14). So, $H_{\mathrm{c}}$ corresponds to the Hamiltonian of free particle in noncommutative phase space with the effective parameter of noncommutativity $\tilde{\eta}$. The influence of noncommutativity on the motion of 
the free particle has been studied [10]. The noncommutative momenta $\tilde{P}_{i}$ which satisfy $(14)$ can be represented as

$$
\begin{aligned}
& \tilde{P}_{1}=\tilde{p}_{1}+\frac{\tilde{\eta}}{2} \tilde{x}_{2}, \\
& \tilde{P}_{2}=\tilde{p}_{2}-\frac{\tilde{\eta}}{2} \tilde{x}_{1},
\end{aligned}
$$

where the coordinates $\tilde{x}_{i}$ and the momenta $\tilde{p}_{i}$ satisfy the ordinary commutation relations

$$
\begin{aligned}
& {\left[\tilde{x}_{1}, \tilde{x}_{2}\right]=0,} \\
& {\left[\tilde{p}_{1}, \tilde{p}_{2}\right]=0,} \\
& {\left[\tilde{x}_{i}, \tilde{p}_{j}\right]=i \hbar .}
\end{aligned}
$$

Therefore, the Hamiltonian $H_{\mathrm{c}}$ can be rewritten as

$$
\begin{aligned}
H_{\mathrm{c}} & =\frac{\tilde{p}_{1}^{2}}{2 M}+\frac{\tilde{p}_{2}^{2}}{2 M}+\frac{\tilde{\eta}^{2}}{8 M}\left(\tilde{x}_{1}^{2}+\tilde{x}_{2}^{2}\right) \\
& -\frac{\tilde{\eta}}{2 M}\left(\tilde{x}_{1} \tilde{p}_{2}-\tilde{x}_{2} \tilde{p}_{1}\right) .
\end{aligned}
$$

Introducing the following operators (see, for example, $[6,11])$

$$
\begin{aligned}
& b_{1}=\frac{1}{2}\left(-i \xi_{1}-i \frac{d}{d \xi_{1}}+\xi_{2}+\frac{d}{d \xi_{2}}\right), \\
& b_{1}^{+}=\frac{1}{2}\left(i \xi_{1}-i \frac{d}{d \xi_{1}}+\xi_{2}-\frac{d}{d \xi_{2}}\right)
\end{aligned}
$$

with

$$
\xi_{1}=\sqrt{\frac{\tilde{\eta}}{2 \hbar}} x_{1}, \quad \xi_{2}=\sqrt{\frac{\tilde{\eta}}{2 \hbar}} x_{2}
$$

we can write

$$
H_{\mathrm{c}}=\frac{\hbar \tilde{\eta}}{M}\left(b_{1}^{+} b_{1}+\frac{1}{2}\right) .
$$

Note that the following relation is satisfied

$$
\left[b_{1}, b_{1}^{+}\right]=1 \text {. }
$$

So, the eigenvalues of $H_{\mathrm{c}}$ read [10]

$$
\begin{array}{r}
E_{n_{1}}^{c}=\hbar \Omega_{1}\left(n_{1}+\frac{1}{2}\right) \\
\Omega_{1}=\frac{\tilde{\eta}}{M}
\end{array}
$$

where $n_{1}$ is a quantum number, $n_{1}=0,1,2,3, \ldots$.

Now let us consider the relative motion which is described by $H_{\text {rel }}$. Note that the coordinates and the momenta of the relative motion satisfy commutation relations (15)-(17) and can be represented as

$$
\begin{aligned}
& \Delta X_{1}=\sqrt{\frac{\Delta \theta \Delta \eta}{2(1-\sqrt{1-\Delta \theta \Delta \eta})}}\left(\Delta x_{1}-\frac{1}{\Delta \eta}(1-\sqrt{1-\Delta \theta \Delta \eta}) \Delta p_{2}\right), \\
& \Delta X_{2}=\sqrt{\frac{\Delta \theta \Delta \eta}{2(1-\sqrt{1-\Delta \theta \Delta \eta})}}\left(\Delta x_{2}+\frac{1}{\Delta \eta}(1-\sqrt{1-\Delta \theta \Delta \eta}) \Delta p_{1}\right), \\
& \Delta P_{1}=\sqrt{\frac{\Delta \theta \Delta \eta}{2(1-\sqrt{1-\Delta \theta \Delta \eta})}}\left(\Delta p_{1}+\frac{1}{\Delta \theta}(1-\sqrt{1-\Delta \theta \Delta \eta}) \Delta x_{2}\right), \\
& \Delta P_{2}=\sqrt{\frac{\Delta \theta \Delta \eta}{2(1-\sqrt{1-\Delta \theta \Delta \eta})}}\left(\Delta p_{2}-\frac{1}{\Delta \theta}(1-\sqrt{1-\Delta \theta \Delta \eta}) \Delta x_{1}\right) .
\end{aligned}
$$

So, the Hamiltonian $H_{\text {rel }}$ can be rewritten as

$$
\begin{aligned}
& H_{\text {rel }}=\left(\frac{\Delta \theta \Delta \eta}{4 \mu(1-\sqrt{1-\Delta \theta \Delta \eta})}+\frac{k \Delta \theta(1-\sqrt{1-\Delta \theta \Delta \eta})}{4 \Delta \eta}\right) \Delta \mathbf{p}^{2} \\
& +\left(\frac{k \Delta \theta \Delta \eta}{4(1-\sqrt{1-\Delta \theta \Delta \eta})}+\frac{\Delta \eta(1-\sqrt{1-\Delta \theta \Delta \eta})}{4 \mu \Delta \theta}\right) \Delta \mathbf{x}^{2}+\left(\frac{\Delta \eta}{2 \mu}+\frac{k \Delta \theta}{2}\right)\left(\Delta x_{1} \Delta p_{2}-\Delta x_{2} \Delta p_{1}\right) .
\end{aligned}
$$

Introducing the set of operators (see, for example, $[6,11]$ )

$$
\begin{aligned}
& b_{2}=\frac{1}{2}\left(-i \tilde{\xi}_{1}-i \frac{d}{d \tilde{\xi}_{1}}+\tilde{\xi}_{2}+\frac{d}{d \tilde{\xi}_{2}}\right), \\
& b_{2}^{+}=\frac{1}{2}\left(i \tilde{\xi}_{1}-i \frac{d}{d \tilde{\xi}_{1}}+\tilde{\xi}_{2}-\frac{d}{d \tilde{\xi}_{2}}\right), \\
& b_{3}=\frac{1}{2}\left(-i \tilde{\xi}_{1}-i \frac{d}{d \tilde{\xi}_{1}}-\tilde{\xi}_{2}-\frac{d}{d \tilde{\xi}_{2}}\right), \\
& b_{3}^{+}=\frac{1}{2}\left(i \tilde{\xi}_{1}-i \frac{d}{d \tilde{\xi}_{1}}-\tilde{\xi}_{2}+\frac{d}{d \tilde{\xi}_{2}}\right),
\end{aligned}
$$


where $\tilde{\xi}_{1}=l_{0} x_{1}, \tilde{\xi}_{2}=l_{0} x_{2}$ with

$$
\begin{aligned}
& l_{0}=\sqrt{\frac{\hbar}{\tilde{\mu} \tilde{\omega}}}, \\
& \frac{1}{\tilde{\mu}}=\frac{\Delta \theta \Delta \eta}{2 \mu(1-\sqrt{1-\Delta \theta \Delta \eta})}+\frac{k \Delta \theta(1-\sqrt{1-\Delta \theta \Delta \eta})}{2 \Delta \eta}, \\
& \tilde{\omega}=\sqrt{\frac{\Delta \theta \Delta \eta}{2 \mu(1-\sqrt{1-\Delta \theta \Delta \eta})}+\frac{k \Delta \theta(1-\sqrt{1-\Delta \theta \Delta \eta})}{2 \Delta \eta}} \\
& \times \sqrt{\left(\frac{k \Delta \theta \Delta \eta}{2(1-\sqrt{1-\Delta \theta \Delta \eta})}+\frac{\Delta \eta(1-\sqrt{1-\Delta \theta \Delta \eta})}{2 \mu \Delta \theta}\right)},
\end{aligned}
$$

the Hamiltonian $H_{\text {rel }}$ reads

$$
H_{\text {rel }}=\hbar \Omega_{2}\left(b_{2}^{+} b_{2}+\frac{1}{2}\right)+\hbar \Omega_{3}\left(b_{3}^{+} b_{3}+\frac{1}{2}\right)
$$

where

$$
\begin{aligned}
& \Omega_{2}=\tilde{\omega}+\left(\frac{\Delta \eta}{2 \mu}+\frac{k \Delta \theta}{2}\right), \\
& \Omega_{3}=\tilde{\omega}-\left(\frac{\Delta \eta}{2 \mu}+\frac{k \Delta \theta}{2}\right) .
\end{aligned}
$$

Note that operators (51)-(54) satisfy the following commutation relations

$$
\begin{aligned}
& {\left[b_{1}, b_{1}^{+}\right]=\left[b_{2}, b_{2}^{+}\right]=1,} \\
& {\left[b_{1}^{+}, b_{2}\right]=\left[b_{1}^{+}, b_{2}^{+}\right]=\left[b_{2}^{+}, b_{1}\right]=\left[b_{2}^{+}, b_{1}^{+}\right]=0 .}
\end{aligned}
$$

So, the eigenvalues of $H_{\text {rel }}$ read

$$
E_{n_{2}, n_{3}}^{\mathrm{rel}}=\hbar \Omega_{2}\left(n_{2}+\frac{1}{2}\right)+\hbar \Omega_{3}\left(n_{3}+\frac{1}{2}\right)
$$

where $n_{2}, n_{3}$ are the quantum numbers $n_{2}=0,1,2,3 \ldots$, $n_{3}=0,1,2,3 \ldots$ and $\Omega_{2}, \Omega_{3}$ are defined by (45), (59), (60). Taking into account (29), (44), (63), we can write the spectrum of a two-particle system with harmonic oscillator interaction in noncommutative phase space

$$
\begin{aligned}
E_{n_{1}, n_{2}, n_{3}} & =E_{n_{1}}^{c}+E_{n_{2}, n_{3}}^{\mathrm{rel}}=\hbar \Omega_{1}\left(n_{1}+\frac{1}{2}\right) \\
& +\hbar \Omega_{2}\left(n_{2}+\frac{1}{2}\right)+\hbar \Omega_{3}\left(n_{3}+\frac{1}{2}\right) .
\end{aligned}
$$

So, because of noncommutativity of momenta the motion of the center-of-mass is not free in noncommutative phase space. The energy levels of $H_{\mathrm{c}}$ are determined by the first two terms in (64). These levels correspond to the energy levels of the harmonic oscillator with the frequency $\tilde{\eta} / M$. The relative motion of the system is described by $H_{\text {rel }}$. The spectrum of the motion corresponds to the spectrum of the two-dimensional harmonic oscillator with frequencies (22), (23). So, the spectrum of a two-particle system with harmonic oscillator interaction corresponds to the spectrum of the tree-dimensional harmonic oscillator with frequencies $\Omega_{1}, \Omega_{2}, \Omega_{3}$ which depend on the effective parameters of noncommutativity $\tilde{\eta}$, $\Delta \theta, \Delta \eta$. Note that in the limits $\theta_{i} \rightarrow 0, \eta_{i} \rightarrow 0, i=(1,2)$ (in these limits, according to (20)-(23), one has $\tilde{\eta} \rightarrow 0$, $\Delta \theta \rightarrow 0, \Delta \eta \rightarrow 0$ ) one obtains the well known result for the spectrum of a two-particle system with harmonic oscillator interaction.

\section{CONCLUSIONS}

In this paper, a space with noncommutativity of coordinates and noncommutativity of momenta (1)-(3) has been studied. We have considered the case when different particles feel noncommutativity with different parameters. In this case, the two-particle problem has been examined. It is worth noting that the motion of the centerof-mass of the system and the relative motion are not independent in the noncommutative phase space (18), (19). In our previous paper [40], it has been shown that in the case when the parameters of noncommutativity which correspond to a particle are determined by its mass as (24), (25), a list of problems is solved in the noncommutative phase space. Among them are the problem of the dependence of the motion of the center-of-mass of a composite system on the relative motion, the problem of the violation of the equivalence principle, the problem of the violation of properties of the kinetic energy. The importance of conditions (24), (25) is stressed by the number of problems which can be solved. Therefore, in this paper we have considered the noncommutative phase space with parameters of noncommutativity determined as $(24),(25)$. In this case the motion of the center-ofmass of a composite system and the relative motion are independent. Therefore, the two-particle problem can be reduced to the problem of one particle in the noncommutative phase space.

A two-particle system with harmonic oscillator inter- 
action has been examined. We have studied the influence of the coordinates noncommutativity and the momenta noncommutativity on the motion of the center-of-mass and the relative motion. We have found exactly the energy levels of the system in the noncommutative phase space (64). These levels correspond to the spectrum of harmonic oscillator with frequences $\Omega_{1}, \Omega_{2}, \Omega_{3}$ (45), (59), (60) which depend on the effective parameters of noncommutativity $\tilde{\eta}, \Delta \theta, \Delta \eta(21)-(23)$.

\section{ACKNOWLEDGMENTS}

This work was supported in part by the European Commission under the project STREVCOMS PIRSES2013-612669 and projects $\Phi \phi-63 H p$ (No. 0117U007190), $\Phi ф-30 \Phi$ (No. 0116U001539) from the Ministry of Education and Science of Ukraine.
[1] R. Jackiw, Ann. Henri Poincarre 4, 913 (2003).

[2] H. Snyder, Phys. Rev. 71, 38 (1947).

[3] N. Seiberg, E. Witten, J. High En. Phys. 9909, 032 (1999).

[4] S. Doplicher, K. Fredenhagen, J. E. Roberts, Phys. Lett. B 331, 39 (1994).

[5] A. Hatzinikitas, I. Smyrnakis, J. Math. Phys. 43, 113 (2002).

[6] A. Kijanka, P. Kosinski, Phys. Rev. D 70, 127702 (2004).

[7] Jing Jian, Jian-Feng Chen, Eur. Phys. J. C 60, 669 (2009).

[8] A. Smailagic, E. Spallucci, Phys. Rev. D 65, 107701 (2002).

[9] A. Smailagic, E. Spallucci, J. Phys. A 35, 363 (2002).

[10] A. E. F. Djemai, H. Smail, Commun. Theor. Phys. 41, 837 (2004).

[11] P. R. Giri, P. Roy, Eur. Phys. J. C 57, 835 (2008).

[12] J. Ben Geloun, S. Gangopadhyay, F. G. Scholtz, Europhys. Lett. 86, 51001 (2009).

[13] D. Nath, P. Roy Ann. Phys, 377, 115 (2017).

[14] Pei-Ming Ho, Hsien-Chung Kao, Phys. Rev. Lett. 88 151602 (2002).

[15] M. Chaichian, M. M. Sheikh-Jabbari, A. Tureanu, Phys. Rev. Lett. 86, 2716 (2001).

[16] M. Chaichian, M. M. Sheikh-Jabbari, A. Tureanu, Eur. Phys. J. C 36, 251 (2004).

[17] O. Bertolami, R. Queiroz, Phys. Lett. A 375, 4116 (2011).

[18] N. Chair, M. A. Dalabeeh, J. Phys. A: Math. Gen. 38, 1553 (2005).

[19] A. Stern, Phys. Rev. Lett. 100, 061601 (2008).

[20] S. Zaim, L. Khodja, Y. Delenda, Int. J. Mod. Phys. A 26, 4133 (2011).

[21] T. C. Adorno, M. C. Baldiotti, M. Chaichian, D. M. Gitman, A. Tureanu, Phys. Lett. B 682, 235 (2009).

[22] L. Khodja, S. Zaim, Int. J. Mod. Phys. A 27, 1250100 (2012).

[23] S. A. Alavi, Mod. Phys. Lett. A 22, 377 (2007).

[24] Kh. P. Gnatenko, V. M. Tkachuk, Phys. Lett. A 378, 3509 (2014).

[25] Kh. P. Gnatenko, Yu. S. Krynytskyi, V. M. Tkachuk,
Mod. Phys. Lett. A 30, 1550033 (2015).

[26] Kh. P. Gnatenko, J. Phys.: Conf. Ser. 670, 012023 (2016).

[27] Kh. P. Gnatenko, V. M. Tkachuk, Int. J. Mod. Phys. A 32, 1750161 (2017).

[28] M. M. Stetsko, V. M. Tkachuk, Phys. Lett. A 372, 5126 (2008).

[29] J. Gamboa, M. Loewe, F. Mendez, J. C. Rojas, Mod. Phys. Lett. A 16, 2075 (2001).

[30] P. A. Horvathy, Ann. Phys. 299128 (2002).

[31] O. F. Dayi, L. T. Kelleyane, Mod. Phys. Lett. A 171937 (2002).

[32] M. Daszkiewicz, Acta Phys. Polon. B 44, 59 (2013).

[33] O. Bertolami, J. G. Rosa, C. M. L. de Aragao, P. Castorina, D. Zappala, Phys. Rev. D 72, 025010 (2005)

[34] C. Bastos, O. Bertolami, Phys. Lett. A 372, 5556 (2008).

[35] J. Gamboa, M. Loewe, J. C. Rojas, Phys. Rev. D 64, 067901 (2001).

[36] J. M. Romero, J. D. Vergara, Mod. Phys. Lett. A 18, 1673 (2003).

[37] B. Mirza, M. Dehghani, Commun. Theor. Phys. 42, 183 (2004).

[38] A. E. F. Djemai, Int. J. Theor. Phys. 43, 299 (2004).

[39] Kh. P. Gnatenko, V. M. Tkachuk, Mod. Phys. Lett. A 31, 1650026 (2016).

[40] Kh. P. Gnatenko, V. M. Tkachuk, Phys. Lett. A 381, 2463 (2017).

[41] Kh. P. Gnatenko, Mod. Phys. Lett. A 32, 1750166 (2017).

[42] M. Daszkiewicz, C. J. Walczyk, Mod. Phys. Lett. A 26, 819 (2011).

[43] Kh. P. Gnatenko, Phys. Lett. A 377, 3061 (2013).

[44] Kh. P. Gnatenko, J. Phys. Stud. 17, 4001 (2013).

[45] M. Daszkiewicz, Acta Phys. Polon. B 44, 699 (2013).

[46] Kh. P. Gnatenko, V. M. Tkachuk, Ukr. J. Phys. 61, 432 (2016)

[47] V. M. Tkachuk, Phys. Rev. A 86, 062112 (2012).

[48] C. Quesne, V. M. Tkachuk, Phys. Rev. A 81, 012106 (2010).

[49] V. M. Tkachuk, Found. Phys. 46, 1666 (2016). 


\title{
СИСТЕМА ДВОХ ЧАСТИНОК З ОЦИЛЯТОРНОЮ ВЗАЕМОДІЕЮ В НЕКОМУТАТИВНОМУ ФАЗОВОМУ ПРОСТОРІ
}

\author{
Х. П. Гнатенко, В. М. Ткачук \\ Кафедра теоретичної фізики, Львівсъкий націоналъний університет імені Івана Франка, \\ вул. Драгоманова, 12, Лъвів, 79005, Україна
}

Систему двох частинок досліджено у просторі з некомутативністю координат та некомутативністю імпульсів. Розглянуто випадок, коли різні частинки відчувають некомутативність із різними параметрами, що визначаються їхньою масою. Знайдено точно спектр двочастинкової системи з осциляторною взаємодією в некомутативному фазовому просторі. 